\title{
Biological therapies in the systemic management of psoriasis: International Consensus Conference
}

\author{
W.STERRY, J.BARKER, ${ }^{*}$ W.-H.BOEHNCKE, $\dagger$ J.D.BOS, + S.CHIMENTI, $\S$ \\ E.CHRISTOPHERS, M.DE LA BRASSINNE, ${ }^{* *}$ C.FERRANDIZ, $\uparrow+$ C.GRIFFITHS, + \% \\ A. KATSAMBAS, $\S$ K.KRAGBALLE, $\uparrow$ C.LYNDE, ${ }^{* * *}$ A.MENTER, ${ }^{*}+\dagger$

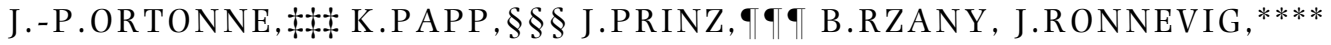 \\ J.-H.SAURAT, $\uparrow+\uparrow \dagger$ M.STAHLE, $+\uparrow+\uparrow$ F.M.STENGEL, $\S \S \S$ \\ P.VAN DE KERKHOF q \& J. VOORHEES***** \\ Department of Dermatology and Allergy, University Hospital Charité, Schumannstr. 20/21, D-10117 Berlin, Germany \\ *St John's Institute of Dermatology, St Thomas' Hospital, London, UK \\ †Johann Wolfgang Goethe-University, Frankfurt am Main, Germany \\ $\ddagger$ Academic Medical Center, University of Amsterdam, Amsterdam, the Netherlands \\ §Clinica Dermatologica, Università degli Studi di Roma 'Tor Vergata', Rome, Italy \\ -University of Kiel, Kiel, Germany \\ **University Hospital Liège, Liège, Belgium \\ † Hospital Universitario Germans Trias i Pujol, Barcelona, Spain \\ **University of Manchester, Manchester, UK \\ $\S \S$ University of Athens Medical School, Athens, Greece \\ - Aarhus University Hospital, Aarhus, Denmark \\ ***Lynde Centre for Dermatology, Toronto, Ontario, Canada \\ t††Texas Dermatology Research Institute, Dallas, TX, U.S.A. \\ †Nice University, Nice, France \\ $\S \S \S$ University of Western Ontario, London, Ontario, Canada \\ ฯฯUniversity of Munich, Munich, Germany \\ ****Department of Dermatology, The National Hospital, Oslo, Norway \\ ††† + Hôpital Cantonal Universitaire, Geneva, Switzerland \\ $\$+\$$ Karolinska University Hospital, Solna, Stockholm, Sweden \\ $\S \S \S \S$ University Institute Cemic Dermatology, Buenos Aires, Argentina \\ $\uparrow \uparrow \uparrow$ University Hospital Nijmegen, Nijmegen, the Netherlands \\ *****University of Michigan Medical School, Ann Arbor, MI, U.S.A.
}

Summary Psoriasis is a chronic, immune-mediated disorder that usually requires long-term treatment for control. Approximately $25 \%$ of patients have moderate to severe disease and require phototherapy, systemic therapy or both. Despite the availability of numerous therapeutic options, the long-term management of psoriasis can be complicated by treatment-related limitations. With advances in molecular research and technology, several biological therapies are in various stages of development and approval for psoriasis. Biological therapies are designed to modulate key steps in the pathogenesis of psoriasis. Collectively, biologicals have been evaluated in thousands of patients with psoriasis and have demonstrated significant benefit with favourable safety and tolerability profiles. The limitations of current psoriasis therapies, the value of biological therapies for psoriasis, and guidance regarding the incorporation of biological therapies into clinical practice are discussed.

Key words: alefacept, biologicals, efalizumab, etanercept, infliximab, psoriasis

Correspondence: Wolfram Sterry

E-mail: wolfram.sterry@charite.de

For conflicts of interest see the Acknowledgements section.

\section{Introduction}

Psoriasis is a chronic immune-mediated disease afflicting approximately $2 \%$ of the Caucasian population. ${ }^{1}$ Psoriasis can be associated with significant physical and psychological morbidity, with an impact on 
physical and mental disability comparable with that of other major medical illnesses such as rheumatoid arthritis, hypertension, heart disease and depression. $^{2,3}$ Various factors limit favourable long-term outcomes with currently available therapies, in particular their lack of consistent efficacy over time, ${ }^{4}$ the risk of serious cumulative toxicity, ${ }^{5}$ and inconvenience.

Approximately one-quarter of all patients with chronic plaque psoriasis require phototherapy, systemic therapy or both to control their disease adequately. ${ }^{6}$ The most frequently used systemic therapies for these patients include ciclosporin, methotrexate, oral retinoids and psoralen plus ultraviolet (UV) A (PUVA), ${ }^{7}$ although the use of narrowband UVB is gradually increasing. While not commonly used in most countries, fumaric acid esters are widely used in Germany, and hydroxyurea (hydroxycarbamide) and sulfasalazine are occasionally used in certain centres.

Although the aetiology and pathogenesis of psoriasis are not fully understood, there is substantial evidence to support the role of the immune system, particularly relating to the roles of $\mathrm{T}$ cells and cytokines. ${ }^{1,8-11}$ Based on the continuous progress in psoriasis research and advances in molecular biology and technology, a new class of agents - targeted biological therapies - has emerged. ${ }^{12}$ These biologicals are designed to block specific molecular steps important in the pathogenesis of psoriasis. In addition, biological therapies have been used and are in development for other therapeutic areas such as Crohn's disease and rheumatoid arthritis. ${ }^{13}$ Currently, three types of biologicals are approved or are in development for psoriasis: (i) recombinant human cytokines, (ii) monoclonal antibodies, and (iii) fusion proteins. ${ }^{13}$ Based on current hypotheses regarding psoriasis immunopathogenesis, two main therapeutic approaches have emerged: modulating either T-cell activities or cytokines (Table 1). ${ }^{14}$ Within these main two approaches, specific strategies being explored include reducing the number of pathogenic $\mathrm{T}$ cells (e.g. CD45RO+ $\mathrm{T}$ cells); inhibiting T-cell activation and trafficking; deviating the immune response (e.g. altering the cytokine balance to favour type 1 vs. type 2 cytokine production); and blocking the activity of proinflammatory cytokines. ${ }^{13,} 14$

Phase I-III clinical trials conducted over the last decade have demonstrated that biologicals provide clinical benefit for the treatment of psoriasis. Using internationally acknowledged safety and efficacy endpoints, the overall utility and benefit of the biologicals has been demonstrated based on the percentage of patients achieving at least a 50\% improvement in
Table 1. Biological therapies for psoriasis

\begin{tabular}{|c|c|}
\hline Agent & Phase \\
\hline \multicolumn{2}{|c|}{ Agents targeting $T$ cells or antigen-presenting cells } \\
\hline Alefacept & Approved for psoriasis in the U.S.A. \\
\hline Efalizumab (anti-CD11a) & $\begin{array}{l}\text { Approved for psoriasis in the U.S.A.; } \\
\text { submitted to the European Agency for } \\
\text { the Evaluation of Medicinal Products }\end{array}$ \\
\hline OKTcdr4a (anti-CD4) & Phase III \\
\hline CTLA4-Ig & Phase I \\
\hline $\begin{array}{l}\text { Denileukin diftitox } \\
\left(\mathrm{DAB}_{389} \text {-IL2) }\right.\end{array}$ & $\begin{array}{l}\text { Phase I trials for psoriasis; approved for } \\
\text { CD25+ cutaneous T-cell lymphoma } \\
\text { in the U.S.A. }\end{array}$ \\
\hline \multicolumn{2}{|l|}{ Agents targeting cytokines } \\
\hline Infliximab (anti-TNF- $\alpha$ ) & $\begin{array}{l}\text { Phase III trials for psoriasis; approved } \\
\text { for Crohn's disease, RA and AS in the } \\
\text { U.S.A. and EU }\end{array}$ \\
\hline Etanercept (anti-TNF- $\alpha$ ) & $\begin{array}{l}\text { Supplemental Biologies License } \\
\text { Application filed in the U.S.A. for } \\
\text { psoriasis; approved for RA, AS, } \\
\text { psoriatic arthritis and juvenile chronic } \\
\text { arthritis in the U.S.A. and EU }\end{array}$ \\
\hline Adalimumab (anti-TNF- $\alpha)$ & $\begin{array}{l}\text { Phase III trials for psoriasis; approved } \\
\text { for RA in the U.S.A. and EU }\end{array}$ \\
\hline IL-10 & Phase II \\
\hline Onercept (anti-TNF- $\alpha$ ) & Phase II \\
\hline Anti-IL-12 & Phase II \\
\hline $\mathrm{IL}-4$ & Phase I \\
\hline IL-11 & Phase I \\
\hline
\end{tabular}

TNF, tumour necrosis factor; IL, interleukin; RA, rheumatoid arthritis; AS, ankylosing spondylitis; EU, European Union.

Psoriasis Area and Severity Index (PASI), a 75\% improvement in PASI (PASI-75), the mean change in PASI over time, the impact of treatment on quality of life (QOL), and safety and tolerability. In clinical trials, efficacy is generally measured in the short term in selected patients whereas effectiveness is considered to be the overall effect achieved in clinical practice. Tolerability, convenience and compliance are important factors that impact on the level of effectiveness. In the absence of a cure for psoriasis, the optimum therapeutic option is one that offers the best ratio between improvement of skin lesions, and inconvenience and toxicity. ${ }^{15}$

Two large patient surveys, one conducted by the National Psoriasis Foundation (NPF) and the other by the European Union Federation of Psoriasis Associations, have highlighted the significant patient dissatisfaction with currently available therapeutic options. ${ }^{16,17}$ The NPF survey revealed that only $18 \%$ of survey respondents with severe psoriasis were currently receiving systemic therapy; $32 \%$ of patients indicated that their psoriasis therapy was not aggressive enough. ${ }^{16}$

With three biologicals (alefacept, efalizumab and etanercept) approved for psoriasis in the U.S.A. and 
four biologicals (adalimumab, anakinra, etanercept and infliximab) that collectively have been used in rheumatology and gastroenterology in more than 700000 patients over several years, it is appropriate to assess the utility of biologicals in the context of daily practice. The International Consensus Conference was convened in order to define the current unmet medical needs of psoriasis, to assess the value of biological therapies in psoriasis management and to provide general guidance regarding the use of these new agents. Although there are variations among the biologicals with respect to efficacy, safety and administration, the purpose of this meeting was not to compare and contrast the individual biological therapies, but rather to consider their attributes as a therapeutic class. Four biologicals-alefacept, efalizumab, etanercept and infliximab-were considered representative of the biologicals given their phase of development (Phase III) or approval and the availability of published data regarding their use in psoriasis.

Twenty-three dermatologists, from Argentina, Belgium, Canada, Denmark, France, Germany, Greece, Italy, the Netherlands, Norway, Spain, Sweden, Switzerland, the U.K. and the U.S.A., convened for a 1-day workshop on 21 January 2004, in Zurich, Switzerland. The discussions facilitated by the chairman (see Acknowledgements) during the Conference were based primarily upon published trial data, as well as on the personal experience of the participants. All major aspects were discussed first in working groups and later in a plenary session. The various consensus points were voted upon and approved; in this manuscript, they appear in italics, preceded by 'Consensus'. This manuscript was developed in accordance with these consensus outputs.

\section{Current systemic anti-psoriatic therapy and unmet medical needs}

\section{Efficacy}

Consensus: Current therapies effectively control symptoms in the short term; however, additional therapies with favourable, long-term safety profiles are needed. As detailed in Table 2, current therapies have good efficacy, reducing the severity and extent of psoriasis. However, the level of evidence for these current therapies varies, as many were approved for psoriasis prior to the standardization of efficacy end-points and without the benefit of controlled clinical studies. Naldi et al. recently highlighted the lack of standardized assessment of efficacy. ${ }^{18}$ Among 171 studies that assessed efficacy using a scoring system for the severity of psoriasis, 44 different scoring systems were used. The assessment of clinical efficacy data for current therapies is furthermore influenced by a number of factors, including variations in entry criteria or baseline characteristics, varying dosages and treatment duration, heterogeneous data, and either inadequate or inconsistent documentation of outcome measures. ${ }^{18-20}$

While there have been several randomized, placebocontrolled trials that evaluated ciclosporin for psoriasis, this is not the case for other therapies that have been used for decades. ${ }^{19}$ The scope, design and control of trials evaluating ciclosporin are considerably smaller ${ }^{19}$ when compared with studies involving biologicals. It is apparent that, in addition to the published literature, assessments of effectiveness are supported by our extensive clinical experience with these modalities. Despite the effectiveness of current psoriasis therapies, there is a medical need for therapies specifically targeted at psoriasis pathogenesis, as opposed to the broader mechanism of action of current systemic therapies. Furthermore, while psoriasis is a life-long disease requiring long-term management, agents that demonstrate rapid clinical response as well as sustained effectiveness are desirable. Finally, given that neither current therapies nor biologicals are curative, cure and/or prevention remain the major unmet medical need for psoriasis.

\section{Safety}

Consensus: The long-term safety profiles of current therapies may limit their continuous use. These long-term safety and tolerability profiles have not been well documented in large, well-designed clinical trials. Although the short-term safety profiles of these agents have been well characterized, their long-term safety profiles in large numbers of patients have not been well defined in clinical trials. Reviews have demonstrated that of the few randomized controlled trials in psoriasis, most are short-term, ${ }^{18,20}$ with a median study duration of 7 weeks. ${ }^{18}$ The long-term safety profiles of these current therapies are therefore derived primarily from clinical experience. For example, methotrexate has been used safely for long periods (e.g. 10-20 years); however, no substantial documentation exists from large numbers of patients. ${ }^{21}$ The long-term use of current modalities is limited by a number of factors, the most important of which is the 


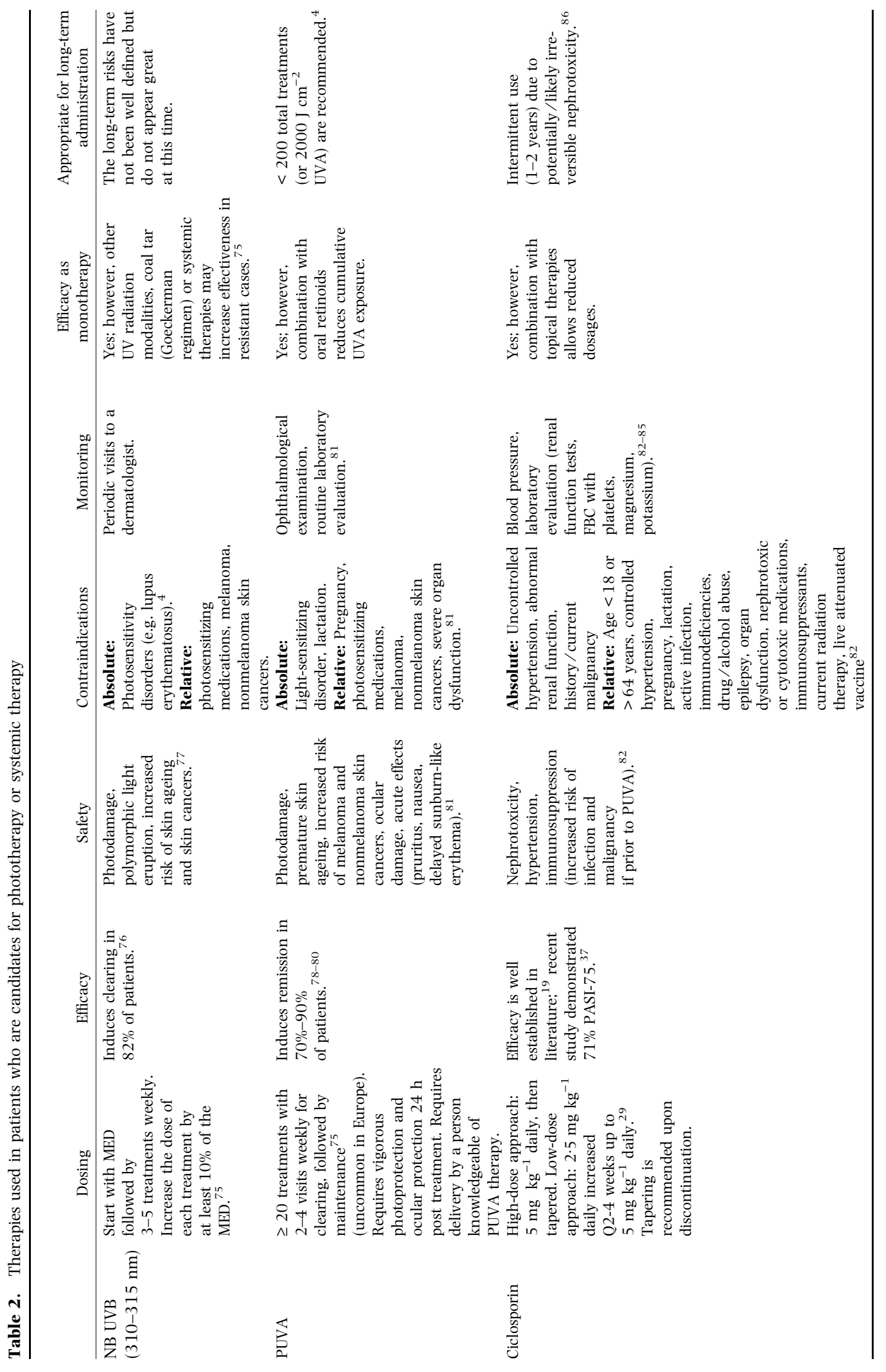




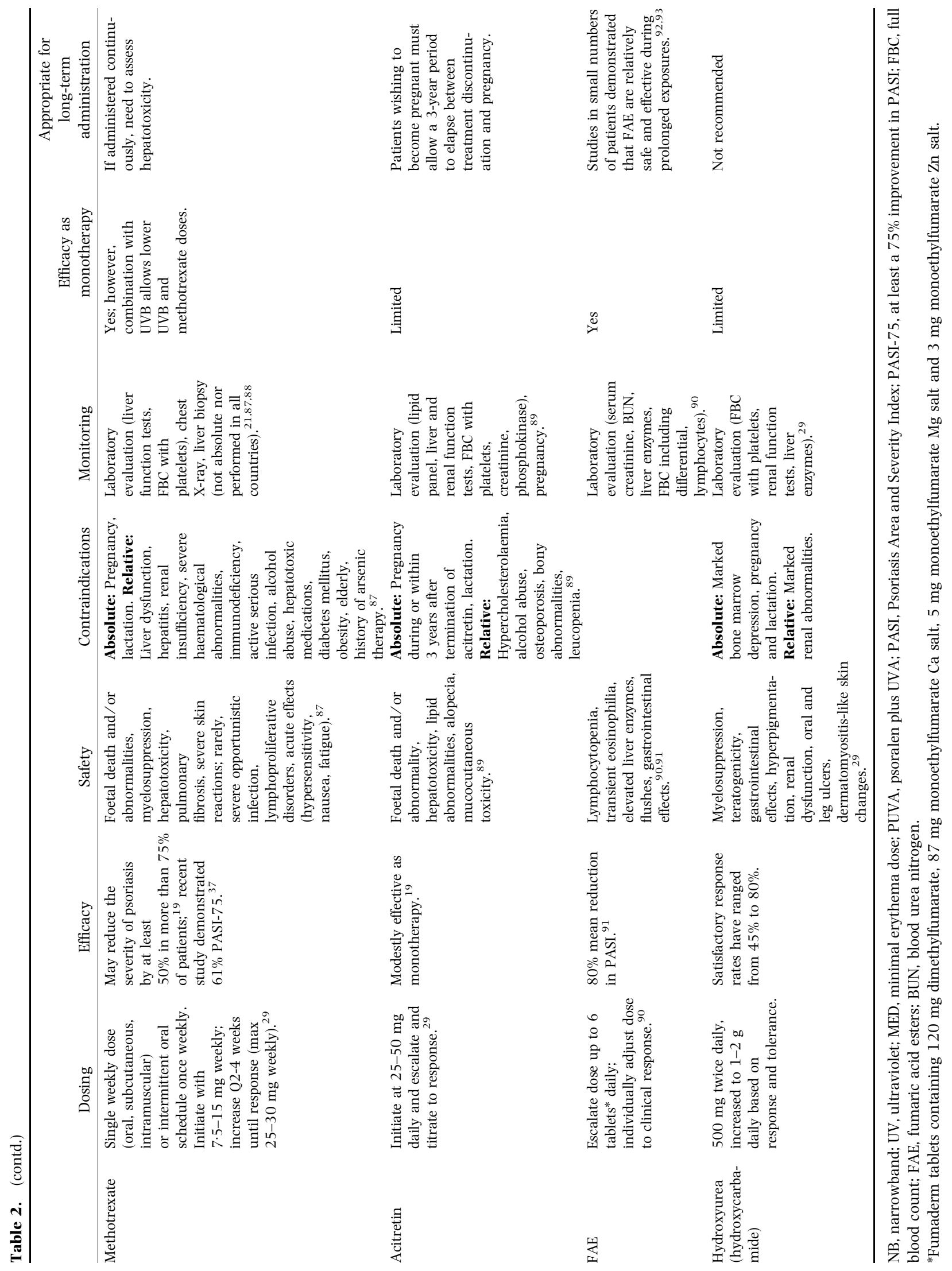


treatment-related toxicity that restricts their ability to be administered on a long-term basis (Table 2). Given the risk of cumulative toxicities associated with ciclosporin, methotrexate and various types of phototherapy, notably end-organ toxicity and malignancy, guidelines have been developed in order to improve their safety during administration and to minimize their toxicity. For example, to minimize the risk of nephrotoxicity associated with ciclosporin, continuous administration beyond 1-2 years is not recommen$\operatorname{ded}^{22}$ In order to detect the risk of hepatotoxicity associated with methotrexate, liver biopsies are recommended (at cumulative dose intervals of $1.5 \mathrm{~g}$ ) in addition to liver function tests. ${ }^{21}$ More recently, the radioimmunoassay of serum levels of the aminopropeptide of collagen III has been recommended for early detection of liver fibrosis in long-term methotrexate therapy. Although some patients are given ciclosporin or methotrexate for prolonged periods, many physicians and patients are hesitant to prescribe or to continue therapy on a long-term basis. ${ }^{23}$ When patients become intolerant of their current therapy, develop concurrent conditions that prohibit the continuation of treatment, or reach maximum cumulative exposure/toxicities, the selection of an alternative therapy is often necessary. ${ }^{24-28}$ Drug-drug interactions with ciclosporin and methotrexate are important safety considerations because they limit therapeutic options for some patients. ${ }^{4}$

Several drugs may potentiate the toxicity of methotrexate through a variety of mechanisms, including alterations in protein binding, decreased renal excretion of methotrexate, and synergistic hepatotoxicity. ${ }^{29}$ Ciclosporin is metabolized by the cytochrome P450 3A system, ${ }^{30}$ the source of the majority of drug-drug interactions. $^{31}$

\section{Treatment of different age groups}

Consensus: Therapies that can be safely administered to patients of all ages and life stages are needed. Most of the current systemic therapeutic options are not desirable for infants and children, who will require long-term therapy over many decades to control their disease. Furthermore, elderly patients with psoriasis are likely to have concomitant illnesses and medications, which complicate therapeutic decisions. As psoriasis presents early in life in the majority of cases, with an equal incidence in males and females, the therapeutic options for women of childbearing age are extremely limited given the teratogenic effects of some therapies, most notably methotrexate and acitretin. Moreover, methotrexate therapy is not advised in men planning to conceive children. Additionally, topical therapies such as corticosteroids may be associated with systemic toxicities (e.g. hypothalamic-pituitary-adrenal axis suppression), which may limit their utility. ${ }^{32}$

\section{Monitoring}

Consensus: The safety profiles of current systemic therapies necessitate frequent or invasive monitoring; treatments with minimal monitoring requirements are preferable. In order to avoid the serious toxicities associated with current therapies, frequent and/or invasive monitoring is necessary (Table 2). Considering the time, economics and inconvenience, these monitoring requirements are important to physicians and patients alike.

\section{Combination therapy}

Consensus: Psoriasis therapies that are effective as monotherapy and provide safe, long-term control are needed. Given variations in effectiveness between individual agents, variations in effectiveness over time and the risk of treatment-related toxicity, various treatment approaches (e.g. combination, rotational, sequential and intermittent therapy) have evolved to address the need for long-term control. ${ }^{24-28}$

Although ciclosporin, methotrexate and PUVA are effective as a monotherapy, combination approaches are often used to allow the administration of reduced dosages in an effort to improve safety. ${ }^{33-35}$ Acitretin monotherapy is modestly effective; therefore, it may be combined with other therapeutic modalities, such as PUVA, to improve efficacy. ${ }^{19}$ Topical therapies are often added to systemic therapies or phototherapy to reduce the dose of the systemic therapy or exposure to phototherapy or to improve efficacy. Although combining various treatment modalities may reduce their toxicity, it is nonetheless important to consider that such approaches may be impractical for some patients. Furthermore, the efficacy and safety of most combination therapies have rarely been subject to a clinical trial.

\section{Convenience}

Consensus: Therapies that are more convenient than current systemic treatments are needed to improve patient 
compliance with treatment, thus improving therapeutic outcomes. The convenience of a given therapeutic approach can be affected by multiple factors, including dosing frequency, route of administration, accessibility, lifestyle, ability to administer monotherapy, time and effort required, and limitations in the ability to administer long-term treatment. While oral therapies are simple and convenient for patients in terms of administration, there are limitations associated with other current therapies. For example, the inconvenience of topical therapies (e.g. messy, odorous, time needed to apply) and phototherapy (e.g. limited accessibility, time commitment) may reduce compliance with the prescribed regimen. Additionally, rotating, sequencing or combining therapies is impractical for some patients. Thus, effective and safe long-term therapies that could reduce the need for combining, rotating or sequencing therapies would be expected to improve convenience and, ultimately, overall compliance.

\section{Impact on quality of life}

Consensus: Both the physical and psychosocial aspects of psoriasis need to be considered and treated; assessments need to capture physical manifestations and psychosocial issues. In addition to assessing the ability of a given therapy to improve psoriasis using objective measures, it is important that the impact of treatment on QOL be considered. Despite the well-recognized adverse impact of psoriasis on QOL, ${ }^{16,17}$ reviewed by Choi \& Koo, ${ }^{36}$ there are few published reports regarding improvements achieved with the use of current therapies. $^{18,37}$ In the review of Naldi et al., a single paper evaluated QOL. ${ }^{18}$ Some of the current therapies, by virtue of the fact that their administration may be impractical or associated with toxic effects, have been shown to have a negative impact on QOL. ${ }^{16,17}$ In summary, given the attributes of current systemic therapies, there is a major unmet medical need for psoriasis therapies that safely and effectively provide psoriasis patients with continuous control of their disease and that have a favourable impact on their QOL (Table 3).

\section{The value of biological therapies for psoriasis management}

The focus of the consensus conference discussion was based on the medicine and the science of biological therapies; the health economic impact was not
Table 3. Consensus: unmet medical needs for psoriasis treatment

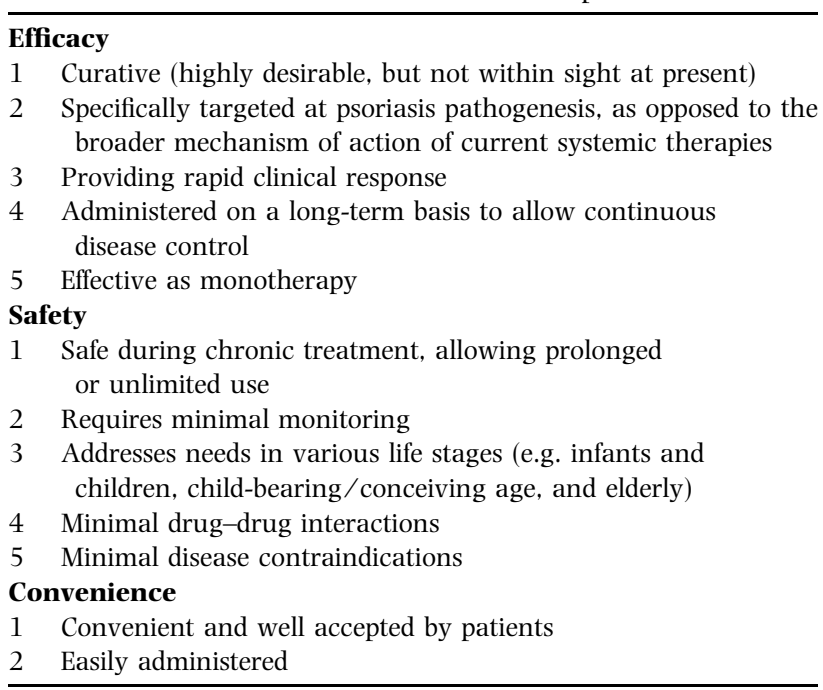

addressed in this particular forum. Biologicals, while a therapeutic class, differ in terms of their mechanisms of action, and efficacy and safety profiles. Such differences will become important when selecting the appropriate therapy for patients on an individual basis. It was the overall value of biologicals that was discussed collectively during this meeting.

\section{Efficacy}

Consensus: As a therapeutic class, biologicals are effective for the treatment of psoriasis. There is robust evidence from multiple, large, well-designed, randomized, placebo-controlled trials of biological therapies for psoriasis (Table 4). Entry criteria for the trials evaluating biologicals for psoriasis varied slightly from study to study but generally included involvement of at least $5 \%{ }^{38}-10 \%$ body surface area ${ }^{39-41}$ and a minimal PASI response of $10^{41}$ to 12 points. ${ }^{40}$ All of the trials for each of these agents met their primary end-points, with a significantly greater proportion of patients who received the biological therapy achieving a PASI-75 response compared with those patients who received placebo (Table 4). Efalizumab, etanercept and infliximab are associated with early clinical response, within 4 weeks of initiating treatment.

Safety

Consensus: Biologicals have been proven relatively safe in the short to intermediate term; longer-term safety and efficacy outcomes will need to continue to be observed and accumulated. Biologicals have proven to be relatively 


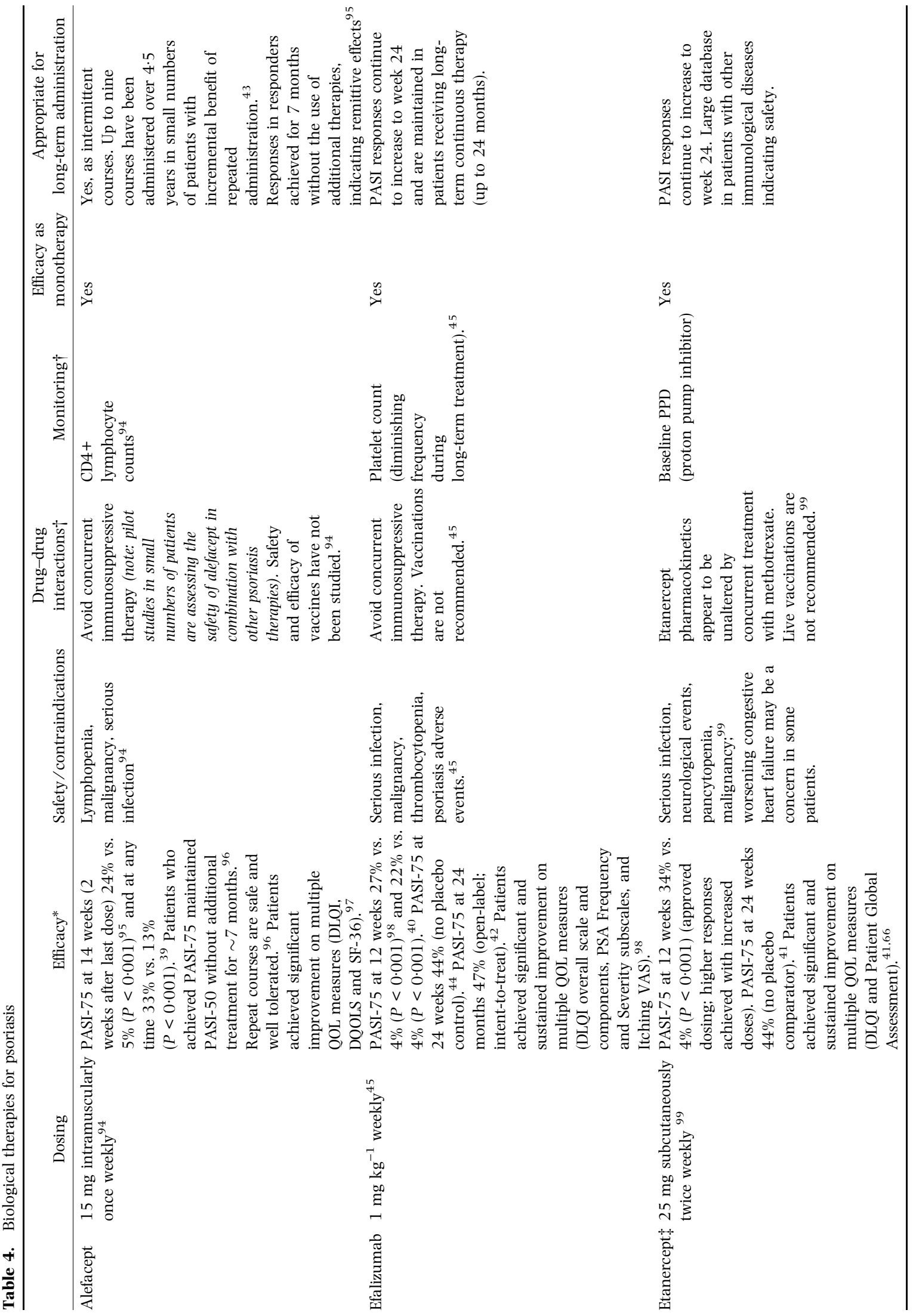




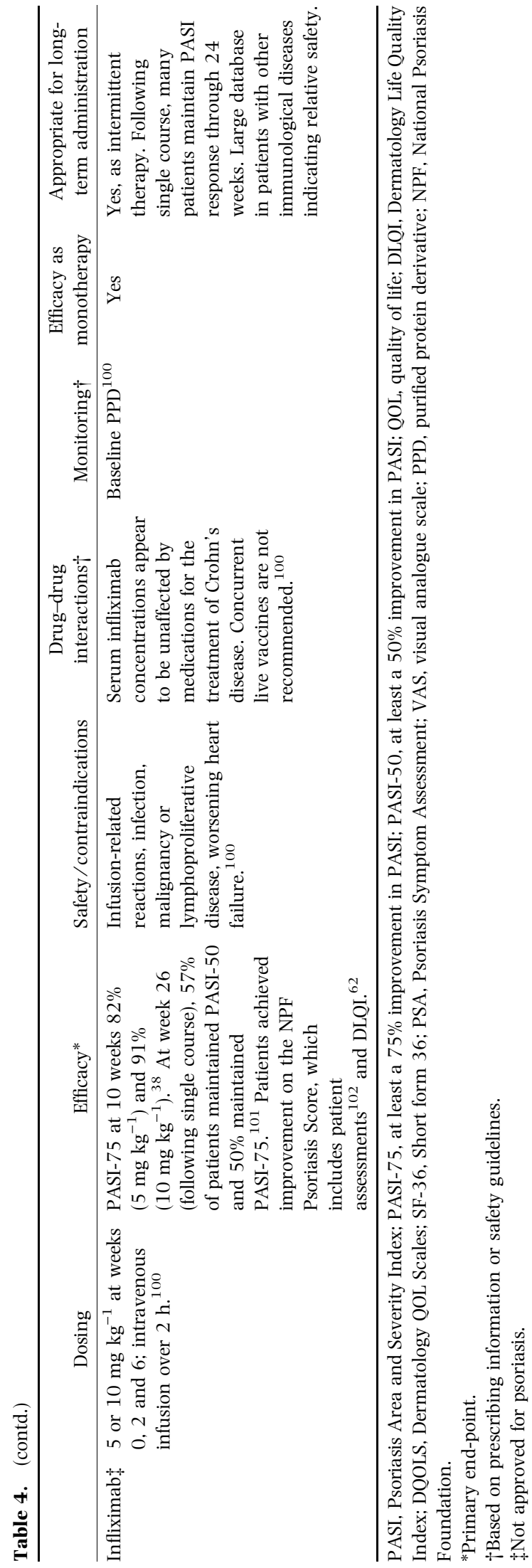

safe during short- and intermediate-term administration (Table 4). The short-term adverse events are mostly benign (e.g. acute influenza-like symptoms upon initiating therapy), but serious infusion reactions may rarely occur. Biological therapies do not appear to show any evidence of hepatotoxicity or nephrotoxicity. Efalizumab and alefacept have been evaluated in patients with psoriasis for periods of $2-4 \cdot 5$ years. ${ }^{42,} 43$ Data available for up to 24 months of continuous efalizumab therapy and up to nine cycles of alefacept therapy indicate that, in addition to sustained efficacy, there is no increase in toxicity over time. ${ }^{42-44}$ Rare cases $(0 \cdot 3 \%)$ of reversible thrombocytopenia have been observed in efalizumab-treated patients during clinical trials. ${ }^{45}$ During alefacept therapy, memory T-cell counts are reduced as a likely consequence of the mechanism of alefacept action, with no significant reduction noted over multiple courses of therapy. ${ }^{46} \mathrm{~A}$ 12-week course of alefacept did not impair primary or secondary antibody responses to a neoantigen, or memory responses to a recall antigen. ${ }^{47}$

There are considerable longer-term safety data for etanercept and infliximab used for diseases other than psoriasis, where they have been proven to be relatively safe therapeutic approaches in the majority of patients. There are several potential concerns with anti-tumour necrosis factor (TNF)- $\alpha$ therapies that have been observed in small numbers of patients with Crohn's disease or rheumatoid arthritis, including infections, antinuclear antibody formation, ${ }^{48}$ drug-induced autoimmune disorders (e.g. drug-induced lupus erythematosus), ${ }^{49,} 50$ heart failure ${ }^{51}$ and nervous system disorders (including demyelinating disorders), as well as serious infusion reactions. ${ }^{52}$ Long-term data in psoriasis patients will be required to define clearly the long-term safety of these therapies in patients with psoriasis.

The primary concern regarding long-term safety of biologicals relates to the risk of immunosuppression, the level of which may be related to the development of infection or malignancy. ${ }^{53}$ Although cases of lymphoma in patients with rheumatoid arthritis receiving TNF- $\alpha$ inhibitors have been reported, a clear causal relationship has not been established; ${ }^{54}$ the Arthritis Advisory Committee of the US Food and Drug Administration concluded that causality of lymphoma in these patients could not be established with certainty. It has been suggested that the rate of lymphoma in patients with psoriasis who are 65 years or older is threefold the rate observed in patients without psoriasis, underscoring the importance of defining the 
baseline rate of lymphoma in this patient population in order to accurately assess the risk of lymphoproliferative diseases associated with immunosuppressive therapies. $^{55}$ Patients who are receiving monoclonal antibody anti-TNF- $\alpha$ therapy are at increased risk of developing infection, specifically active tuberculosis. ${ }^{56,57}$ Although there may be variations in risk among the different anti-TNF- $\alpha$ inhibitors, the potential risk needs to be considered, as cases have been reported in patients receiving infliximab, etanercept and adalimumab. ${ }^{56,58}$ To be cautious and to minimize the risk, a tuberculin skin test should be performed prior to initiating anti-TNF- $\alpha$ therapy using monoclonal antibodies, with chest radiography as indicated. ${ }^{56,59}$

Although it will be important to understand the impact of these biological therapies on the immune system in the long term and in a larger number of patients with psoriasis, the data to date in other patient populations (e.g. rheumatology, Crohn's disease) are reassuring. ${ }^{60,61}$ However, until the impact is more clearly defined, psoriasis patients treated with biologicals should be carefully observed.

Biological therapeutics are not metabolized by the cytochrome $\mathrm{P} 450$ system; thus, there are no pharmacokinetic drug interactions. Although formal drug interaction studies have not yet been performed, there is no evidence to suggest that biologicals are limited by drug-drug interactions (Table 4). There are fewer disease contraindications for biological therapies than for current systemic therapies (Table 4). Thus, biological therapies may be appropriate for a broader range of patients than are the current systemic therapies; these issues are being explored further in pilot studies in small numbers of patients.

\section{Monitoring and convenience}

Consensus: There are fewer monitoring requirements for biological therapies than for current systemic therapies. Compared with the monitoring requirements for current systemic therapies, there are fewer monitoring requirements prior to initiating and during biological therapy (Table 4). Monitoring requirements for the biological therapies vary among the individual agents, and the recommendations contained within the prescribing information should be followed. Studies have demonstrated that despite the fact that biologicals are injectable, most patients are comfortable with such administration and it does not adversely impact their QOL. ${ }^{62,}{ }^{63}$ Furthermore, the ability of patients to selfadminister many of the biological agents at home obviates the need for frequent visits to the clinic, and particularly for extensive disease, allows severe psoriasis to be managed on an out-patient basis.

\section{Monotherapy and combination therapy with biologicals}

Consensus: Biological therapies are comparably or more effective than current therapies when administered as monotherapy. All biological therapies met their primary efficacy end-point when administered as monotherapy in randomized, placebo-controlled trials. There are relatively few published data regarding the safety of combination therapy with biologicals; however, evidence to date does not indicate that biological therapies exacerbate the toxicity of other psoriasis therapies. ${ }^{48,64}$ Trials of biologicals in other indications, e.g. Crohn's disease, have demonstrated that biologicals do not exacerbate the toxicity of other therapies used for psoriasis, such as methotrexate. ${ }^{48}$ Interim results in small numbers of patients suggest that alefacept can be safely combined with other psoriasis therapies (e.g. high-potency topicals, methotrexate, ciclosporin, oral retinoids and UVB). ${ }^{65}$ Thus, combining biologicals with other immunomodulating therapies can be considered on a case-by-case basis, particularly when tapering patients off current therapies such as methotrexate or ciclosporin. Until appropriate studies have been conducted to confirm their safety and efficacy, biological therapies themselves should not be combined with each other. Combining biologicals with topical therapies is anticipated to be safe; therefore, topical therapies can be added if necessary.

\section{Long-term therapy}

Consensus: Given the apparent lack of traditional endorgan toxicity (e.g. nephrotoxicity or hepatotoxicity), biologicals may he used for significant periods of time. Data to date demonstrate that biological therapies are capable of providing long-term disease control (Table 4). While alefacept is not indicated for continuous long-term administration, the remittive effects, lasting up to 7 months in responders, provide patients with long-term control. Multiple courses of alefacept have been administered in a small number of patients with apparent efficacy and safety. ${ }^{43}$ Efalizumab has demonstrated sustained efficacy without increased toxicity during continuous dosing of up to 24 months. ${ }^{42}$ As in rheumatoid arthritis and Crohn's disease, long-term administration of etanercept and 
long-term intermittent use of infliximab appear to be feasible for psoriasis, though neither agent is currently approved for such use in psoriasis. Data and experience suggest that biologicals promise to provide psoriasis patients with long-term control of their disease.

\section{Impact on quality of life}

Consensus: In addition to improving the physical signs of psoriasis, there is ample evidence from large randomized, controlled trials to demonstrate that biological therapies improve multiple facets of quality of life. Patients demonstrated significant improvement in QOL as determined by multiple measures, including Dermatology QOL Scales, Short form 36 and Dermatology Life Quality Index (Table 4). Improvement on these patient-reported scales reflects improved functionality (e.g. ability to perform job or attend school), decreased impact of treatment on daily living, improved social relations, and a reduction in the overall frequency and severity of psoriasis symptoms. Importantly, the improvements achieved during shortterm administration (e.g. 12 weeks) were sustained with extended dosing. ${ }^{44,66}$

The new treatment paradigm: consensus guidance for treatment of psoriasis

Consensus: Treatment of psoriasis no longer requires a strict, step-wise approach; instead, decisions can be based on patient presentation, disease severity and patient-specific characteristics. Treatment decisions are based upon a variety of factors, including the extent and site of involvement, type of psoriasis, burden of disease and/or disability, prior psoriasis treatments (including effectiveness, cumulative doses if applicable, and tolerance), age and life stages, pregnancy considerations, concomitant illnesses or medications, extent of disability, the patient's goals and expectations from therapy, and the overall convenience of the therapeutic regimen and the patient's ability to comply with the prescribed treatment.

For many clinicians, psoriasis management has typically followed a progression in which patients fail the previous 'step' before treatment with a more aggressive (and more toxic) therapy is initiated; therefore, treatment considerations in psoriasis typically progress sequentially from topical therapies to phototherapy and finally to systemic therapy. ${ }^{4,20,67}$ However, topical therapy may not be considered as initial therapy in patients with severe psoriasis. Current practice patterns using biologicals are providing new support for the notion that psoriasis management does not require a strict, step-wise approach.

In 2003, the American Academy of Dermatology developed a consensus statement for the treatment of moderate to severe plaque psoriasis. ${ }^{68}$ According to that statement, biological therapies can be considered alongside current systemic therapies in patients who are candidates for systemic therapy. Based on efficacy and safety profiles, convenience and QOL improvement achieved with biological therapies, it was agreed that biologicals should be given equal consideration among primary agents that are appropriate in patients who are candidates for systemic therapy. Many of the participants favoured biological therapies over current systemic agents for the treatment of chronic plaque psoriasis in certain situations, based on available safety data at this relatively early time in the clinical experience with biologicals.

Our suggested approach for integrating biologicals into clinical practice is illustrated in Figure 1. Various instances in which biologicals should be considered, from both patient and physician perspectives, are outlined in Table 5. For example, in addition to patients for whom topical therapies are ineffective or impractical and who are candidates for or who have failed or are intolerant to systemic therapy, there are specific instances in which biological therapies are likely to provide therapeutic benefit. Examples include patients for whom current therapies are impractical, patients who are concerned about safety (short- vs. long-term safety), patients with recalcitrant psoriasis or alternate psoriasis morphologies (as discussed below), and patients who have a significant reduction in QOL or who are physically incapacitated. In addition to patient-related factors, physicians should consider biologicals when they wish to prescribe a single agent that can be safely administered, have particular safety concerns or are considering utilising biologicals in combination with acceptable agents.

\section{Future applications}

There is possibly an opportunity to treat forms of psoriasis other than chronic plaque psoriasis, including difficult-to-treat psoriasis, with biologicals. Published data and case reports suggest that biologicals may be as useful as current systemic therapies for recalcitrant psoriasis, ${ }^{69}$ and other subtypes such as palmoplantar pustulosis, erythrodermic psoriasis and pustular psoriasis. $^{70-74}$ 


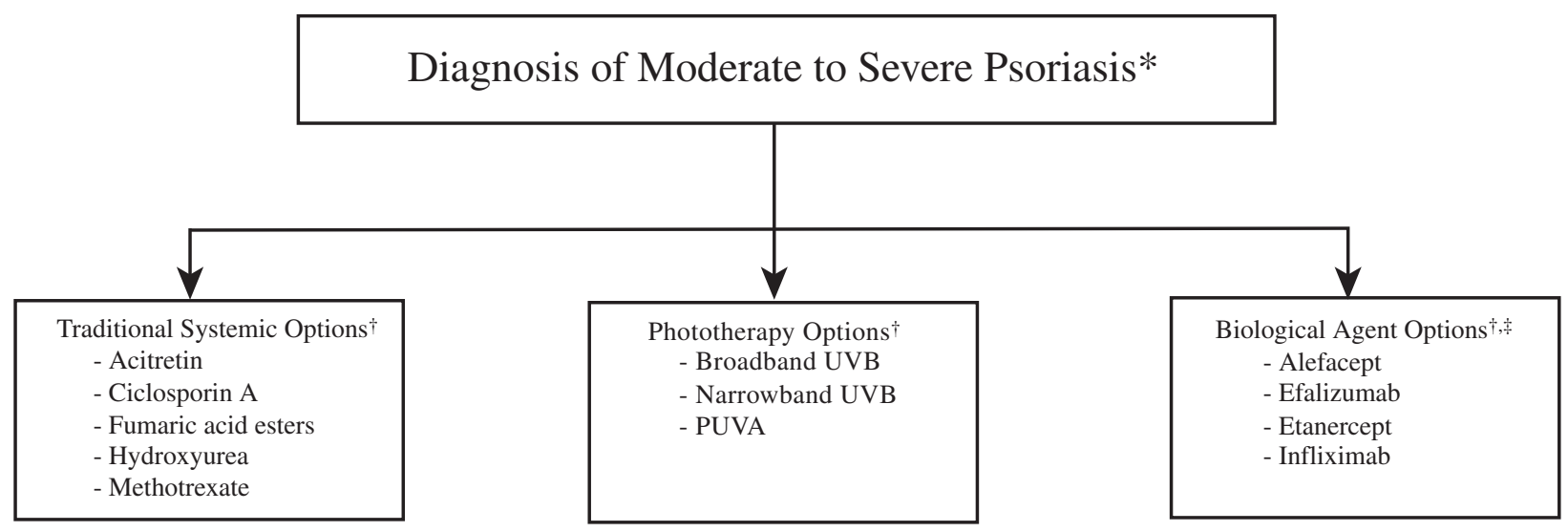

*Moderate to severe psoriasis is understood to be a disease for which systemic therapy is appropriate, A diagnosis of plaque psoriasis is typically based on characteristic appearance; skin biopsies are rarely necessary given expert dermatological care. ${ }^{103,104}$ Given the variable course of psoriasis in terms of location, extent of involvement and associated symptoms, ${ }^{105}$ continuity of care is important in psoriasis management. Definitions of psoriasis severity have not been standardized, ${ }^{105}$ and traditional measures of disease severity are based upon the extent of body surface area involvement. However, such measures fail to consider a variety of factors, such as severe but localized involvement, location of disease (e.g. face, palms or soles and genitals), associated symptoms and impact on quality of life. ${ }^{105,106}$ Thus, disease severity is considered on a case-by-case basis.

$\dagger$ Each of the agents within each category may be an equivalent option, based on disease- and patient-specific factors. Combinations of various treatment options are possible; however, patient and drug-specific factors must be considered for each case.

$\$$ Reflects agents with published data to support their use in psoriasis; as favourable data evolve for additional agents, they would be appropriate for consideration.

Figure 1. Consensus: incorporating biological therapies into clinical practice. ${ }^{103-106}$

Table 5. Consensus: instances where biological therapies should be considered

Patient-related considerations

1 Patients for whom topical therapy is ineffective or impractical

2 Patients with plaque psoriasis who are candidates for systemic therapy

3 Patients who have failed or are intolerant of current systemic therapies

4 Patients for whom current systemic therapies are contraindicated

5 Patients for whom current systemic therapies or phototherapy is impractical (e.g. due to distance to phototherapy treatment facility)

6 Patients with recalcitrant psoriasis

7 Patients with severe impairment of quality of life and/or physical or psychosocial disability

8 Patients who are physically incapacitated (i.e. unable to use topical or ultraviolet therapy)

Physician-related considerations

1 Case-based need for practical monotherapy options

2 Case-based need for a long-term therapeutic option

3 Particular safety concerns

4 Case-based requirements for biologicals in combination with other psoriasis therapies, or transition with other psoriasis therapies (this requires further study and may involve multiple combinations)

While cost must be considered an important factor in treatment decisions, our discussions focused on clinical and scientific aspects. It is important that dermatologists advocate the best therapies possible and advise regula- tory bodies and agencies regarding the value of such therapies. This is of particular relevance in a chronic disease such as psoriasis, which has significant QOL as well as physical issues for patients.

\section{Conclusions}

Dermatologists need to familiarize themselves with these new therapies, particularly as regards rationale, their mechanisms of action, and most importantly, efficacy and safety in the treatment of psoriasis. Furthermore, dermatologists should have the opportunity to use and prescribe biological therapies for their patients as their colleagues in rheumatology and gastroenterology have done for the past 5 years. Given the diverse morphologies and fluctuating nature of psoriasis, as well as the psychosocial impact of the disease, dermatologists are in a better position than other specialists to manage the complexities of psoriasis on a long-term basis. Available evidence shows that biological therapies provide shortterm, and perhaps offer long-term control of psoriasis, coupled with improved safety, tolerability, convenience and improvement in QOL. This is the beginning of an exciting era for dermatologists and patients alike. Biologicals appear to offer great promise in the day-today and longer-term management of patients with psoriasis and hopefully will allow the dermatology 
profession to take its rightful place alongside other medical subspecialties using biological therapy.

\section{Acknowledgements}

The International Consensus Conference convened in Zurich, Switzerland, on 21 January 2004, under the sponsorship of Serono International S.A. Serono International S.A. did not participate in the workshop or influence the output in any way. Professor Wolfram Sterry, Department of Dermatology and Allergy, University Hospital Charité, Berlin, Germany, served as the chairman. Assistance in the preparation of this manuscript was provided by Kirsten M. Duncan, PharmD, and Donna L. Schroeder, PharmD.

The following conflicts of interest are declared:

W. Sterry: Grant/research support from 3M, L'Oréal, Fumapharm, Riemser, Schering; consultant for Biogen, Schering, Schering-Plough, Serono, Wyeth.

S. Chimenti: Consultant for Biogen-Dompè.

C. Griffiths: Grant/research support from Novartis, Biogen, Serono, Amgen, Centocor; consultant for Novartis, Serono, Schering-Plough, Wyeth.

K. Kragballe: Grant/research support from Biogen, Centocor, Fumapharm, Genmab, Leo Pharma, Novo Nordisk.

C. Lynde: Grant/research support from Genentech, Xoma, Amgen, Centocor, Serono, GlaxoSmithKline, Allergan, Novartis, Isotechnica, Boehringer-Ingelheim, Leo Pharma, Fujisawa, Biogen; consultant for Genentech, Xoma, Centocor, Serono, Novartis.

A. Menter: Grant/research support from and consultant for Abbott, Amgen, Biogen, Centocor, Genentech, Serono.

J.P. Ortonne: Grant/research support from Biogen, Serono, Novartis; consultant for Abbott.

K. Papp: Grant/research support from Genentech, Xoma, Amgen, Centocor, Abbott, Serono, GlaxoSmithKline, Allergan, Novartis, Isotechnica, Boehringer-Ingelheim, Leo Pharma, Fujisawa, Biogen, Medimmune, Genetics Institute; consultant for Genentech, Xoma, Amgen, Centocor, Serono, Allergan, Hoffmann LaRoche, Novartis.

J. Prinz: Grant/research support from Biogen; consultant for Biogen, Serono, Wyeth, Essex, Hermal.

B. Rzany: Grant/research support from Fumedica, Serono, Leo Pharma, Schering, Biogen.

F.M. Stengel: Consultant for Serono.

P. van de Kerkhof: Grant/research support from Biogen, Amgen, 3M, Fumapharm, Barrier Therapeutics, Leo Pharma.
J. Voorhees: Grant/research support from Amgen, Abbott Laboratories, Centocor.

\section{References}

1 Prinz J. The role of T cells in psoriasis. J Eur Acad Dermatol Venereol 2003; 17: 257-70.

2 de Arruda LH, De Moracs AP. The impact of psoriasis on quality of life. Br J Dermatol 2001; 144(Suppl. 58): 33-6.

3 Rapp SR, Feldman SR, Exum ML, et al. Psoriasis causes as much disability as other major medical diseases. J Am Acad Dermatol 1999; 41: 401-7.

4 Ashcroft DM, Li Wan Po A, Griffiths CE. Therapeutic strategies for psoriasis. J Clin Pharm Ther 2000; 25: 1-10.

5 Tristani-Firouzi P, Krueger GG. Efficacy and safety of treatment modalities for psoriasis. Cutis 1998; 61: 11-21.

6 Boehncke WH. Immunomodulatory drugs for psoriasis. $\mathrm{Br}$ Med J 2003; 327: 634-5.

7 Mrowietz U. Advances in systemic therapy for psoriasis. Clin Exp Dermatol 2001; 26: 362-7.

8 Bos JD, De Rie MA. The pathogenesis of psoriasis: immunological facts and speculations. Immunol Today 1999; 20: 40-6.

9 Griffiths CE. The immunological basis of psoriasis. J Eur Acad Dermatol Venereol 2003; 17(Suppl. 2): 1-5.

10 Krueger JG. The immunologic basis for the treatment of psoriasis with new biologic agents. J Am Acad Dermatol 2002; 46: 1-23.

11 Nickoloff BJ. The immunologic and genetic basis of psoriasis. Arch Dermatol 1999; 135: 1104-10.

12 Gottlieb AB, Bos JD. Recombinantly engineered human proteins: transforming the treatment of psoriasis. Clin Immunol 2002; 105: 105-16.

13 Singri P, West DP, Gordon KB. Biologic therapy for psoriasis: the new therapeutic frontier. Arch Dermatol 2002; 138: 657-63.

14 Kirby B, Griffiths CE. Novel immune-based therapies for psoriasis. Br J Dermatol 2002; 146: 546-51.

15 Dubertret L. Strategy for treatment of psoriasis: systemic treatments. J Dermatol 1998; 25: 788-92.

16 Krueger G, Koo J, Lebwohl M, et al. The impact of psoriasis on quality of life: results of a 1998 National Psoriasis Foundation patient-membership survey. Arch Dermatol 2001; 137: 280-4.

17 Salonen SH. The EUROPSO psoriasis patient study. treatment history and satisfaction reported by 17,990 members of European psoriasis patient associations. Poster presented at the Spring Symposium of the European Academy of Dermatology and Venereology, 27 February-1 March 2003, Malta.

18 Naldi L, Svensson A, Diepgen T, et al. Randomized clinical trials for psoriasis 1977-2000: the EDEN survey. J Invest Dermatol 2003; 120: 738-41.

19 Griffiths CE, Clark CM, Chalmers RJ, et al. A systematic review of treatments for severe psoriasis. Health Technol Assess 2000; 4: 1-125.

20 Spuls PI, Witkamp L, Bossuyt PM, et al. A systematic review of five systemic treatments for severe psoriasis. Br J Dermatol 1997; 137: 943-9.

21 Roenigk HH Jr, Auerbach R, Maibach H, et al. Methotrexate in psoriasis: consensus conference. J Am Acad Dermatol 1998; 38: 478-85.

22 Lebwohl M, Ellis C, Gottlieb A, et al. Cyclosporine consensus conference: with emphasis on the treatment of psoriasis. $J \mathrm{Am}$ Acad Dermatol 1998; 39: 464-75.

23 Finlay AY. Psoriasis from the patient's point of view. Arch Dermatol 2001; 137: 352-3.

24 Koo JY. Current consensus and update on psoriasis therapy: a perspective from the U.S. J Dermatol 1999; 26: 723-33. 
25 Menter MA, Abramovits W. Rational, sequential and combination regimens in the treatment of psoriasis. Dermatol Ther 1999; 11: $88-95$.

26 Roenigk HH Jr. Combination and rotational therapy for psoriasis. In: Psoriasis (Hrah M, ed.), 3rd edn. New York: Marcel Dekker, 1998: 587-92.

27 van de Kerkhof PC. Therapeutic strategies: rotational therapy and combinations. Clin Exp Dermatol 2001: 26: 356-61.

28 Weinstein GD, White GM. An approach to the treatment of moderate to severe psoriasis with rotational therapy. J Am Acad Dermatol 1993; 28: 454-9.

29 Yamauchi PS, Rizk D, Kormeili T, et al. Current systemic therapies for psoriasis: where are we now? J Am Acad Dermatol 2003; 49: $S 66-77$.

30 Lampen A, Christians U, Bader A, et al. Drug interactions and interindividual variability of ciclosporin metabolism in the small intestine. Pharmacology 1996; 52: 159-68.

31 Li AP, Maurel P, Gornez-Lechon MJ, et al. Preclinical evaluation of drug-drug interaction potential: present status of the application of primary human hepatocytes in the evaluation of cytochrome P450 induction. Chem Biol Interact 1997; 107: 5-16.

32 Lebwohl M, Ali S. Treatment of psoriasis. Part 1. Topical therapy and phototherapy. J Am Acad Dermatol 2001; 45: 487-98.

33 Grossman RM, Thivolet J, Claudy A, et al. A novel therapeutic approach to psoriasis with combination calcipotriol ointment and very low-dose cyclosporine: results of a multicenter placebocontrolled study. J Am Acad Dermatol 1994; 31: 68-74.

34 Paul BS, Momtaz K, Stern RS, et al. Combined methotrexateultraviolet B therapy in the treatment of psoriasis. J Am Acad Dermatol 1982; 7: 758-62.

35 Saurat JH, Geiger JM, Amblard P, et al. Randomized double-blind multicenter study comparing acitretin-PUVA, etretinate-PUVA and placebo-PUVA in the treatment of severe psoriasis. Dermatologica 1988; 177: 218-24.

36 Choi J, Koo JY. Quality of life issues in psoriasis. J Am Acad Dermatol 2003; 49: S57-61.

37 Heydendael VM, Spuls PI, Opmeer BC, et al. Methotrexate versus cyclosporine in moderate-to-severe chronic plaque psoriasis. N Engl J Med 2003; 349: 658-65.

38 Chaudhari U, Romano P, Mulcahy LD, et al. Efficacy and safety of infliximab monotherapy for plaque-type psoriasis: a randomised trial. Lancet 2001; 357: 1842-7.

39 Lebwohl M, Christophers E, Langley R, et al. An international, randomized, double-blind, placebo-controlled phase 3 trial of intramuscular alefacept in patients with chronic plaque psoriasis. Arch Dermatol 2003; 139: 719-27.

40 Lebwohl M, Tyring SK, Hamilton TK, et al. A novel targeted T-cell modulator, efalizumab, for plaque psoriasis. N Engl J Med 2003; 349: 2004-13.

41 Leonardi CL, Powers JL, Matheson RT, et al. Etanercept as monotherapy in patients with psoriasis. N Engl J Med 2003; 349: 2014-22.

42 Gottlieb AB, Gordon KB, Caro I, et al. Long-term efalizumab therapy safely maintains psoriasis area and severity index improvement: preliminary results from an open-label trial. Poster presented at the 62nd Annual Meeting of the American Academy of Dermatology, 6-11 February 2004: Washington, DC. Poster 611.

43 Menter A, Cather JC. Long-term use of alefacept: safety and offtreatment responses in patients who have received multiple courses of therapy. Poster presented at the 62nd Annual Meeting of the American Academy of Dermatology, 6-11 February 2004: Washington, DC. Poster 608.

44 Gordon KB, Siegfried E, Carey W, et al. Impact of 24 weeks of continuous efalizumab therapy on patient-reported outcomes in patients with moderate to severe plaque psoriasis. Poster presented at the Meeting of the American Association of Dermatology Academy, 25-29 July 2003: Chicago, IL.

45 Genentech Inc. Raptiva ${ }^{\mathrm{TM}}$ (efalizumab). South San Francisco, CA: Genentech Inc., October 2003 (Package insert).

46 Cooper JC, Morgan G, Harding S, et al. Alefacept selectively promotes NK cell-mediated deletion of CD45RO+ human T cells. Eur J Immunol 2003; 33: 666-75.

47 Gottlieb AB, Casale TB, Frankel E, et al. CD4+ T-cell-directed antibody responses are maintained in patients with psoriasis receiving alefacept: results of a randomized study. J Am Acad Dermatol 2003; 49: 816-25.

48 Baert F, Noman M, Vermeire S, et al. Influence of immunogenicity on the long-term efficacy of infliximab in Crohn's disease. N Engl J Med 2003; 348: 601-8.

49 Debandt M, Vittecoq O, Descamps V, et al. Anti-TNF-alphainduced systemic lupus syndrome. Clin Rheumatol 2003; 22: 56-61.

50 Vermeire S, Noman M, van Assche G, et al. Autoimmunity associated with anti-tumor necrosis factor alpha treatment in Crohn's disease: a prospective cohort study. Gastroenterology 2003; 125: 32-9.

51 Kwon HJ, Cote TR, Cuffe MS, et al. Case reports of heart failure after therapy with a tumor necrosis factor antagonist. Ann Intern Med 2003; 138: 807-11.

52 Mohan N, Edwards ET, Cupps TR, et al. Demyelination occurring during anti-tumor necrosis factor alpha therapy for inflammatory arthritides. Arthritis Rheum 2001; 44: 2862-9.

53 Weinstein GD, Jeffes E, McCullough JL. Cytotoxic and immunologic effects of methotrexate in psoriasis. J Invest Dermatol 1990; 95: $\mathrm{S} 49-52$.

54 Brown SL, Greene MH, Gershon SK, et al. Tumor necrosis factor antagonist therapy and lymphoma development: twenty-six cases reported to the Food and Drug Administration. Arthritis Rheum 2002; 46: 3151-8.

55 Gelfand JM, Berlin J, van Voorhees A, et al. Lymphoma rates are low but increased in patients with psoriasis: results from a population-based cohort study in the United Kingdom. Arch Dermatol 2003; 139: 1425-9.

56 Gardam MA, Keystone EC, Menzies R, et al. Anti-tumour necrosis factor agents and tuberculosis risk: mechanisms of action and clinical management. Lancet Infect Dis 2003; 3: 148-55.

57 Keane J, Gershon S, Wise RP, et al. Tuberculosis associated with infliximab, a tumor necrosis factor alpha-neutralizing agent. N Engl J Med 2001; 345: 1098-104.

58 Wolfe F, Michaud K, Anderson J, et al. Tuberculosis infection in patients with rheumatoid arthritis and the effect of infliximab therapy. Arthritis Rheum 2004; 50: 372-9.

59 Gomez-Reino JJ, Cannona L, Valverde VR, et al. Treatment of rheumatoid arthritis with tumor necrosis factor inhibitors may predispose to significant increase in tuberculosis risk: a multicenter active-surveillance report. Arthritis Rheum 2003; 48: 2122-7.

60 Moreland LW, Cohen SB, Baurngartner SW, et al. Long-term safety and efficacy of etanercept in patients with rheumatoid arthritis. J Rheumatol 2001; 28: 1238-44.

61 Schaible TF. Long term safety of infliximab. Can J Gastroenterol 2000; 14(Suppl. C): C29-32.

62 Feldman SR, Bala M, Menter A, Gordon KB. The quality of life of patients with severe psoriasis treated with infliximab. Poster presented at the 62nd Annual Meeting of the American Academy of Dermatology, 6-11 February 2004: Washington, DC: Poster 7.

63 Papp KA, Miller B, Lynde C, et al. Efalizumab (anti-CD11a) retreatment: final results from an open-label study. Poster presented 
at the Meeting of the American Academy of Dermatology, 21-26 March 2003: San Francisco, CA.

64 Iyer S, Yamauchi P, Lowe NJ. Etanercept for severe psoriasis and psoriatic arthritis: observations on combination therapy. $\mathrm{Br} J$ Dermatol 2002; 146: 118-21.

65 Krueger GG, van de Kerkhof P. Safety of multiple courses of alefacept in combination with other psoriasis therapies: a study that reflects the clinical practice setting. Poster presented at the 62nd Annual Meeting of the American Academy of Dermatology, 6-11 February 2004: Washington, DC. Poster 591.

66 Gottlieb AB, Matheson RT, Lowe N, et al. A randomized trial of etanercept as monotherapy for psoriasis. Arch Dermatol 2003; 139: $1627-32$.

67 Spuls Pl, Bossuyt PM, van Everdingen JJ, et al. The development of practice guidelines for the treatment of severe plaque form psoriasis. Arch Dermatol 1998; 134: 1591-6.

68 Callen JP, Krueger GG, Lebwohl M, et al. AAD consensus statement on psoriasis therapies. J Am Acad Dermatol 2003; 49: 1-4.

69 Gach JE, Berth-Jones J. Successful treatment of recalcitrant psoriasis with a combination of infliximab and hydroxyurea. J Dermatol Treat 2003; 14: 226-8.

70 Elewski BE. Infliximab for the treatment of severe pustular psoriasis. J Am Acad Dermatol 2002; 47: 796-7.

71 Newland MR, Weinstein A, Kerdel F. Rapid response to infliximab in severe pustular psoriasis, von Zumbusch type. Int J Dermatol 2002; 41: 449-52.

72 Rongioletti F, Borenstein M, Kirsner R, et al. Erythrodermic, recalcitrant psoriasis: clinical resolution with infliximab. J Dermatol Treat 2003; 14: 222-5.

73 Spuls PI, Hadi S, Rivera L, et al. Retrospective analysis of the treatment of psoriasis of the palms and soles. J Dermatol Treat 2003; 14(Suppl. 2): 21-5.

74 Weinberg JM. Successful treatment of recalcitrant palmoplantar psoriasis with etanercept. Cutis 2003; 72: 396-8.

75 Zanolli M. Phototherapy treatment of psoriasis today. J Am Acad Dermatol 2003; 49: S78-86.

76 Walters IB, Burack LH, Coven TR, et al. Suberythemogenic narrow-band UVB is markedly more effective than conventional UVB in treatment of psoriasis vulgaris. J Am Acad Dermatol 1999; 40: 893-900.

77 CaIzavara-Pinton PG, Carlino A, Manfredi E, et al. Ocular side effects of PUVA-treated patients refusing eye sun protection. Acta Derm Venereol (Stockh) 1994; 186(Suppl.): 164-5.

78 Lauharanta J. Photochemotherapy. Clin Dermatol 1997; 15: 769-80.

79 Morison WL, Baughman RD, Day RM, et al. Consensus workshop on the toxic effects of long-term PUVA therapy. Arch Dermatol 1998; 134: 595-8.

80 Stem RS. The risk of melanoma in association with long-term exposure to PUVA. J Am Acad Dermatol 2001; 44: 755-61.

81 ICN Pharmaceuticals Inc. Oxsoralen-Ultra ${ }^{\circledR}$ capsules (methoxsalen capsules, USP, $10 \mathrm{mg}$ ). Costa Mesa, CA: ICN Pharmaceuticals Inc., 1998 (package insert).

82 Novartis Pharmaceutical Corp. Neoral ${ }^{\circledR}$ soft gelatin capsules (cyclosporin capsules, USP) modified; Neoral ${ }^{\circledR}$ oral solution (cyclosporin oral solution, USP) modified. East Hanover, NJ: Novartis Pharmaceutical Corp., August 2002 (package insert).

83 De Rie MA, Bos JD. Cyclosporine immunotherapy. Clin Dermatol 1997; 15: 811-21.

84 Gawkrodger M, on behalf of the Therapy Guidelines and Audit Subcommittee of the British Association of Dermatologists. Current management of psoriasis. J Dermatol Treat 1997; 8: 27-55.
85 Lebwohl M. Psoriasis. Lancet 2003; 361: 1197-204.

86 Grossman RM, Chevret S, Abi-Rached J, et al. Long-term safety of cyclosporine in the treatment of psoriasis. Arch Dermatol 1996; 132: 623-9.

87 Lederle Pharmaceutical. Methotrexate sodium tablets, methotrexate sodium for injection, methotrexate $L P F^{\circledR}$ sodium (methotrexate sodium injection) and methotrexate sodium injection. Pearl River, NY: Lederle Pharmaceutical, August 2001 (package insert).

88 Said S, Jeffes EW, Weinstein GD. Methotrexate. Clin Dermatol 1997; 15: 781-97.

89 Roche Laboratories Inc. Soriatane ${ }^{\circledR}$ (acitretin) capsules. Nutley, NJ: Roche Laboratories Inc., 1998 (Package insert).

90 Mrowietz U, Christophers E, Altmeyer P. Treatment of severe psoriasis with fumaric acid esters: scientific background and guidelines for therapeutic use. The German Furnaric Acid Ester Consensus Conference. Br J Dermatol 1999; 141: 424-9.

91 Mrowietz U, Christophers E, Altmeyer P. Treatment of psoriasis with furnatic acid esters: results of a prospective multicentre study. German Multicentre Study. Br J Dermatol 1998; 138: 456-60.

92 Hoefnagel JJ, Thio HB, Willemze R, et al. Long-term safety aspects of systemic therapy with fumaric acid esters in severe psoriasis. $\mathrm{Br}$ J Dermatol 2003; 149: 363-9.

93 Kolbach DN, Nieboer C. Fumaric acid therapy in psoriasis. results and side effects of 2 years of treatment. J Am Acad Dermatol 1992; 27: 769-71.

94 Biogen Inc. Amevive ${ }^{\circledR}$ (alefacept). Cambridge, MA: Biogen Inc., 2003 (Package insert).

95 Ortonne JP. Clinical response to alefacept: results of a phase 3 study of intramuscular administration of alefacept in patients with chronic plaque psoriasis. J Eur Acad Dermatol Venereol 2003; 17(Suppl. 2): 12-6.

96 Gordon KB, Langley RG. Remittive effects of intramuscular alefacept in psoriasis. J Drugs Dermatol 2003; 2: 624-8.

97 Ellis CN, Mordin NM, Adler EY. Effects of alefacept on healthrelated quality of life in patients with psoriasis: results from a randomized, placebo-controlled phase II trial. Am J Clin Dermatol 2003; 4: 131-9.

98 Gordon KB, Papp KA, Hamilton TK, et al. Efalizuamb for patients with moderate to severe plaque psoriasis: a randomized controlled trial. J Am Med Assoc 2003; 290: 3073-80.

99 Immunex Corp. Enbrel ${ }^{\circledR}$ (etanercept). Thousand Oaks, CA: Immunex Corp., 2003 (Package insert).

100 Centocor Inc. Remicade $^{\circledR}$ (infliximab). Malvern, PA: Centocor Inc., 2002 (Package insert).

101 Gottlieb AB, Chaudhari U, Mulcahy LD, et al. Infliximab monotherapy provides rapid and sustained benefit for plaque-type psoriasis. J Am Acad Dermatol 2003; 48: 829-35.

102 Gottlieb AB, Chaudhari U, Baker DG, et al. The National Psoriasis Foundation psoriasis score (NPF-PS) system versus the psoriasis area severity index (PASI) and physician's global assessment (PGA): a comparison. J Drugs Dermatol 2003; 3: 260-6.

103 Gottlieb AB, Psoriasis. Dis Manag Clin Outcomes 1998; 1: 195-202.

104 Pardasani AG, Feldman SR, Clark AR. Treatment of psoriasis: an algorithm-based approach for primary care physicians. Am Fam Physician 2000; 61: 725-33.

105 Krueger GG, Feldman SR, Camisa C, et al. Two considerations for patients with psoriasis and their clinicians: what defines mild, moderate, and severe psoriasis? What constitutes a clinically significant improvement when treating psoriasis? J Am Acad Dermatol 2000; 43: 281-5.

106 Baughman RD, Sobel R. Psoriasis. A measure of severity. Arch Dermatol 1970; 101: 390-5. 